\title{
Prostaglandin E-Major Urinary Metabolite as a Biomarker for Inflammation in Ulcerative Colitis: Prostaglandins Revisited
}

\author{
Yoshinori Arai $^{a}$ Tomokazu Matsuura $^{b}$ Masaaki Matsuura ${ }^{c}$ \\ Mutsunori Fujiwara $^{d}$ Isao Okayasu ${ }^{e}$ Satoru Ito ${ }^{f}$ Seiji Arihiro ${ }^{a}$ \\ ${ }^{a}$ Division of Gastroenterology and Hepatology, Department of Internal Medicine, ${ }^{b}$ Department of Laboratory \\ Medicine, The Jikei University School of Medicine, 'Teikyo University Graduate School of Public Health, \\ ${ }^{\mathrm{d}}$ Department of Clinical Pathology, Japanese Red Cross Medical Center, ${ }^{e}$ Department of Pathology, Kitasato \\ University School of Medicine, and ${ }^{\mathrm{f} I D A C}$ Theranostics, Inc., Tokyo, Japan
}

\section{Key Words}

Ulcerative colitis · Biomarker · Prostaglandin E-major urinary metabolite $\cdot$ Mucosal healing $\cdot$ Tissue remodeling · Colitis-associated cancer

\begin{abstract}
With the development of new therapeutic approaches, the ultimate goal of ulcerative colitis (UC) treatment is not only clinical remission but also mucosal healing. Successful mucosal healing has been associated with a dramatic risk reduction in UC recurrence and colitis-associated cancer development, which are the most critical complications of UC. However, invasive tests such as colonoscopy and biopsy are required to evaluate mucosal healing. Therefore, frequent examinations are unsuitable for UC patients. Mucosal inflammation of the colon and prostaglandin E2 production are assumed to be correlated; therefore, we considered that prostaglandin E-major urinary metabolite (PGE-MUM; 7-hydroxy-5,11-diketotetranor-prosta-1,16-dioic acid) may be a surrogate biomarker of UC activity. In this review, we propose that PGE-MUM levels reflect the colonoscopic and histological appearance of UC, suggesting that it is a more sensitive biomarker than those previously utilized for UC-relat-
\end{abstract}

ed mucosal inflammation. According to the 'organ-specific chronic inflammation-carcinoma sequence' theory, by measuring PGE-MUM periodically, it would be possible to control inflammation, with subsequent prevention of $U C$ recurrence and colitis-associated cancer development. The measurement of urine samples for PGE-MUM - a simple, noninvasive method - can reduce the patient burden as well as medical costs, suggesting its potential for application in routine practice.

c) 2016 S. Karger AG, Basel

\section{Introduction}

The most clinically important complications of ulcerative colitis (UC) are relapse and colitis-associated cancer. Long durations of UC lead to an increased risk of carcinogenesis. The cumulative cancer frequency is $2 \%$ at 10 years, $8 \%$ at 20 years, and $18 \%$ at 30 years [1]. To prevent colitis-associated cancer, long-term continuous inhibition of inflammation is essential $[1,2]$. The evaluation of histological inflammation requires colonoscopic biopsy, which is an invasive technique and therefore impractical for repeated examinations. Instead, examina-

\section{KARGER}

E-Mail karger@karger.com www.karger.com/dig
C 2016 S. Karger AG, Basel

$0012-2823 / 16 / 0931-0032 \$ 39.50 / 0$
Yoshinori Arai and Seiji Arihiro

Division of Gastroenterology and Hepatology, Department of Internal Medicine The Jikei University School of Medicine

3-25-8 Nishi-shimbashi, Minato-ku, Tokyo 105-8461 (Japan)

E-Mail yoshinori.a1@gmail.com and sarihiro@gmail.com 
tion of several biomarkers that reflect the severity of mucosal inflammation has been utilized as a routine method for the assessment of inflammation. The most common biomarker used is C-reactive protein (CRP); however, some reports have observed that even in patients with active UC, CRP levels may stay in the normal range or show only slight changes [3]. Therefore, identifying a new biomarker with superior sensitivity and specificity for UC activity is highly desirable.

A correlation between colon inflammation and prostaglandin E2 (PGE2) production has been reported [4]. Fujiwara et al. [5] reported that in the active phase of a patient with UC, the prostaglandin E-major urinary metabolite (PGE-MUM) levels were significantly higher than those in the remission phase. Previously, we demonstrated that the (PGE-MUM; 7-hydroxy-5,11-diketotetranor-prosta-1,16dioic acid) reflects the histological severity of inflammation in UC [6]. Based on the 'organ-specific chronic inflammation-carcinoma sequence' theory [24], we considered that periodical measurement of PGE-MUM and long-term maintenance of its levels below a certain limit along with appropriate anti-inflammation therapy may reduce UC recurrence and prevent colitis-associated cancer. In this review, we discussed the following points: (i) PGE2 synthesis and metabolism, (ii) PGE2 role in mucosal inflammation and colitis-associated cancer progression, (iii) overall characteristics of PGE-MUM, and (iv) the practical applications of PGE-MUM in the clinical setting.

\section{UC Pathogenesis and the Synthesis and Metabolism of PGE2}

UC is an inflammatory bowel disease with an unknown cause affecting mucosal tissue with erosion and ulcers. Inherited factors together with microenvironmental factors that lead to altered immunity in the intestine have been implicated in the pathogenesis of UC $[7,8]$. Monocytes and macrophages induce abnormal immune reactions through the involvement of cytokines, eicosanoids, and free radicals. Intestinal microflora also affects the immune system in the host, suggesting that it is an important factor affecting UC inflammatory targets under biological conditions. Intestinal microflora affects epithelial gene expression via epigenetic mechanisms as well as intestinal immunity, leading to inflammation. Butyric acid produced by microflora upregulates the Foxp3 gene, leading to regulatory $\mathrm{T}$-cell differentiation that reduces intestinal inflammation [9]. Further, Bifidobacterium longum has been shown to reduce intestinal inflam-

PGE-MUM as a Biomarker for Inflammation in UC mation in a mouse UC model [10]. Some commensal gut bacteria invade mucosal epithelial cells and produce interleukin-8, leading to neutrophil motility and alteration of these areas into active lesions [11]. Moreover, certain anaerobic bacteria produce high concentrations of butyric acid, leading to p53 dependent-apoptotic reactions that cause mucosal erosion [12]. Various reports have described the direct correlation of microbiome modulation and colon tumorigenesis as well as inflammation $[13,14]$.

In the active UC phase, the stimulation of inflammatory cytokines, such as tumor necrosis factor- $\alpha$, leads to the upregulation of cyclooxygenase-2 (COX-2) leading to PGE2 secretion in mucosal tissue. In blood, PGE2 is immediately metabolized by 15 -hydroxy prostaglandin dehydrogenase (15-PGDH), which is present in the lung and colon, into 15-keto-PGE2. Next, in the liver and kidney, 15-ketoPGE2 is converted into 13,14-dihydro-15-keto PGE2 by the action of $\Delta 13$-reductase, followed by $\beta$-oxidation and $\omega$-oxidation; this is finally converted to PGE-MUM (7a-hydroxy-5,11-diketotetranor-prosta-1,16-dioic acid) and excreted along with urine [15].

\section{Role of PGE2 in Mucosal Inflammation}

PGE2 produced during mucosal inflammation prevents absorption of electrolytes such as sodium and chloride, induces vascular hyperpermeability, and enhances bowel movements, leading to diarrhea and bloody feces [16]. Further, PGE2 inhibits neutrophil motility and leukotriene B4 (LTB4) production, with a cytoprotective effect on mucosal epithelium, which suggests its role in mucosal protection [17]. In UC patients, the administration of non-steroidal anti-inflammatory drugs (NSAIDs) worsens intestinal inflammation and does not lead to PGE2 production via cyclooxygenase (COX) inhibition. This adverse effect occurs because NSAIDs induce COX inhibition, leading to the upregulation of the lipoxygenase system, with enhanced production of LTB4 and worsening of intestinal inflammation [4]. Thus, intestinal inflammation in the development and persistence of UC is caused by a complex system of arachidonic acid metabolites, including PGE2 and LTB4.

\section{Role of PGE2 in Carcinogenesis}

Among various prostaglandins, PGE2 plays the most critical role in carcinogenesis. Increased PGE2 levels have been observed in colorectal cancer tissue. The 
pathogenic role of PGE2 in colorectal cancer reportedly involves DNA methylation, leading to silencing of tumor suppressor genes and DNA repair genes [18]. Thereafter, tumor cell growth with inhibition of apoptosis, invasion, and upregulation of angiogenesis leads to carcinogenesis. PGE2 levels are controlled by a balance between its synthetic (COX-2) and metabolic (15PGDH) enzymes. In colorectal cancer tissues, COX-2 expression is upregulated and 15-PGDH expression is reduced, compared with their levels in the normal colon [19]. Increased COX-2 expression is an early phase of carcinogenesis; therefore, NSAIDs are administered to reduce the risk of colorectal cancer and prevent cancer development [20]. Following NSAID therapy, the risk of colorectal cancer is lower in patients with a higher expression of 15-PGDH - considered to be a tumorsuppressor gene - compared with those with low 15$P G D H$ expression [21]. Considering these points, the quantitative measurement of PGE2 is expected to be useful for the prediction of colorectal cancer development.

\section{Colitis-Associated Cancer: ‘Organ-Specific Chronic Inflammation-Carcinoma Sequence'}

Colitis-associated cancer is one of the most important complications in the long-term management of UC. The duration of UC inflammation is an important risk factor for the development of colitis-associated cancer $[1,2]$. Okayasu et al. [22, 23] reported that multiple, repeated inflammation-repair cycles in a mouse UC model with the administration of dextran sulfate sodium leads to dysplasia and carcinoma development, suggesting that long-term successive inflammation is an essential factor for carcinogenesis. With respect to the relationship between chronic inflammation and carcinogenesis, tumor occurrence is more common in organs that experience tissue remodeling caused by chronic inflammation. This concept of 'organ-specific chronic inflammation-carcinoma sequence' has been observed in several organs [24].

The 'chronic hepatitis-cirrhosis-hepatocellular carcinoma sequence' is also a well-known phenomenon. In chronic hepatitis, stellate cells are activated and converted into myofibroblasts (MFB) that produce extracellular matrix (such as collagen) and transforming growth factor- $\beta$, leading to fibrosis (remodeling) in hepatic tissue [25]. In hepatic fibrosis, inflammation stimulates carcinogenesis. The stellate cell is characterized by the expression of $\alpha$-smooth muscle actin and cytoglobin (which turned out to be a suppressor gene) [26, 27]. In UC, significant alterations in cytoglobin and $\alpha$-smooth muscle actin expression are observed in subepithelial MFB, corresponding to colonic stellate cells similar to those observed in cirrhosis [28]. Interferon therapy in hepatitis $\mathrm{C}$ patients has been shown to reduce the occurrence of hepatocellular carcinoma by inhibiting inflammation [29]. Further, hepatocellular carcinoma is more common in hepatitis $\mathrm{C}$ patients with higher alanine transaminase (ALT) levels compared with those with lower ALT levels, while those who maintain ALT levels $\leq 80 \mathrm{IU} / \mathrm{l}$ are less likely to have development and recurrence of cancer [30].

A significant correlation between morphological remodeling in UC with disease duration in the rectal mucosa and neoplastic development has been reported (fig. 1) [31], and p53 and other mutations in the crypt cellular level have been demonstrated in rectal mucosa with remodeling $[32,33]$. Further, the correlation of carcinogenesis with dysfunction of cross-talk between rectal mucosal crypt and interstitial cells has been reported [34, 35]. Along with increased numbers of interstitial MFB instead of reduced numbers of subepithelial MFB, genome dysfunction in epithelial cells and interstitial MFB is critical in carcinogenesis $[34,35]$. Stimulatory inflammation with remodeling leads to a high risk of carcinogenesis [34, 35]. Moreover, colonoscopic inflammation and histological inflammation are both risk factors for colitis-associated cancer. Therefore, strict monitoring and tight control of mucosal inflammation may potentially prevent colitis-associated cancer development.

\section{PGE-MUM Assay}

Alkaline treatment followed by neutralization of the urine sample using a radioimmunoassay kit is a simple measurement method for PGE-MUM levels. The characteristics of this kit have been reported $[6,15]$. This treatment gives rise to a more stable compound for measurement.

\section{Fluctuation of PGE-MUM Levels in Healthy Subjects}

Okayasu et al. [15] reported that PGE-MUM levels in normal healthy subjects $(n=797)$ showed sex- and agerelated differences. PGE-MUM levels in men were higher than those in women. Further, PGE-MUM levels in 


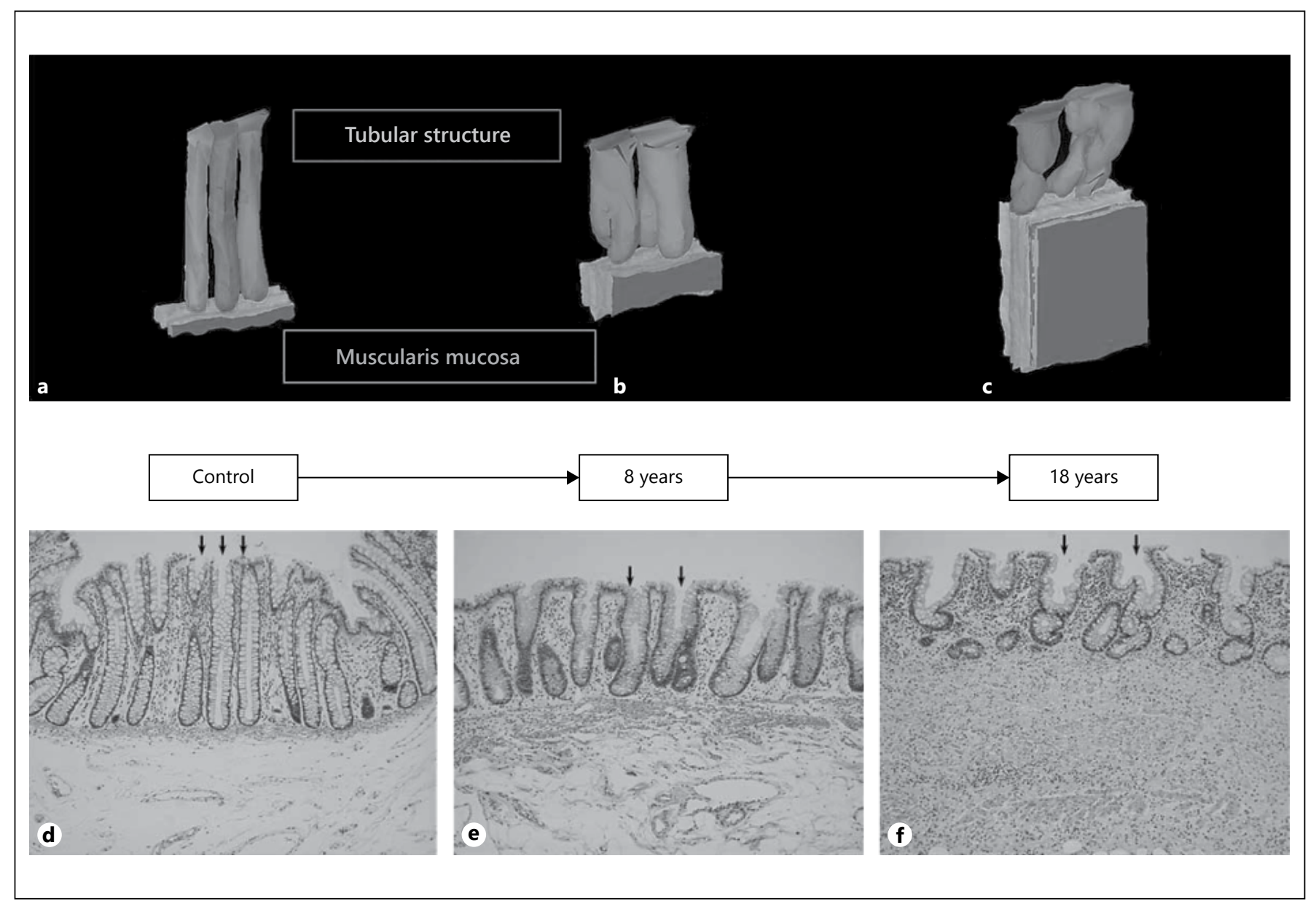

Fig. 1. Mucosal remodeling correlate with UC duration (modified images from [31]). Three-dimensional reconstructed images (a control case without UC; b UC duration, 8 years; $\mathbf{c}$ UC duration, 18 years). Micrograph (d control case without UC; e UC duration, 8 years; f UC duration, 18 years). Repeated or continuous colitis induces tissue destruction and regeneration of the colonic mucosa,

men decreased with age, while levels in women tended to increase. However, among individuals aged over 61, no differences were noted between men and women. This difference was attributed to the effects of estrogen, which may inhibit PGE2 production. Moreover, current smokers had higher PGE-MUM levels than non-smokers and past smokers. This finding suggests that PGEMUM could reflect mild lung inflammation caused by smoking.

Fujiwara et al. [5] reported that laxatives upregulated PGE2 production, leading to increased PGE-MUM levels. However, no statistically significant correlation between PGE-MUM levels and alcoholic consumption has been observed thus far.

PGE-MUM as a Biomarker for Inflammation in UC resulting in remodeling. Small arrows indicate reconstructed crypts. Three-dimensional analyses revealed the crypts to be shortened, curved, and fused with each other, with the extent depending on the duration of UC illness. The muscularis mucosa is thickened. Advanced reconstruction of colonic mucosa is similar to that observed in cirrhosis in chronic hepatitis.

\section{Usefulness of PGE-MUM for the Evaluation of UC Activity}

A correlation between PGE-MUM and UC activity has been reported in a clinical study $(n=99)$ [6]. The evaluation of the simple clinical colitis activity index (SCCAI; $\mathrm{n}=99$ ) [36], Mayo endoscopic scoring (Mayo; $\mathrm{n}=79$ ) [37], and Matts grading (Matts; $n=64$ ) [38] was compared with PGE-MUM and CRP levels, revealing that PGE-MUM and CRP both reflect UC activity $(p<0.01$ for both).

For SCCAI, Mayo, and Matts scoring, PGE-MUM showed higher areas under the curve in the receiver operating characteristic curve than CRP (PGE-MUM vs. 


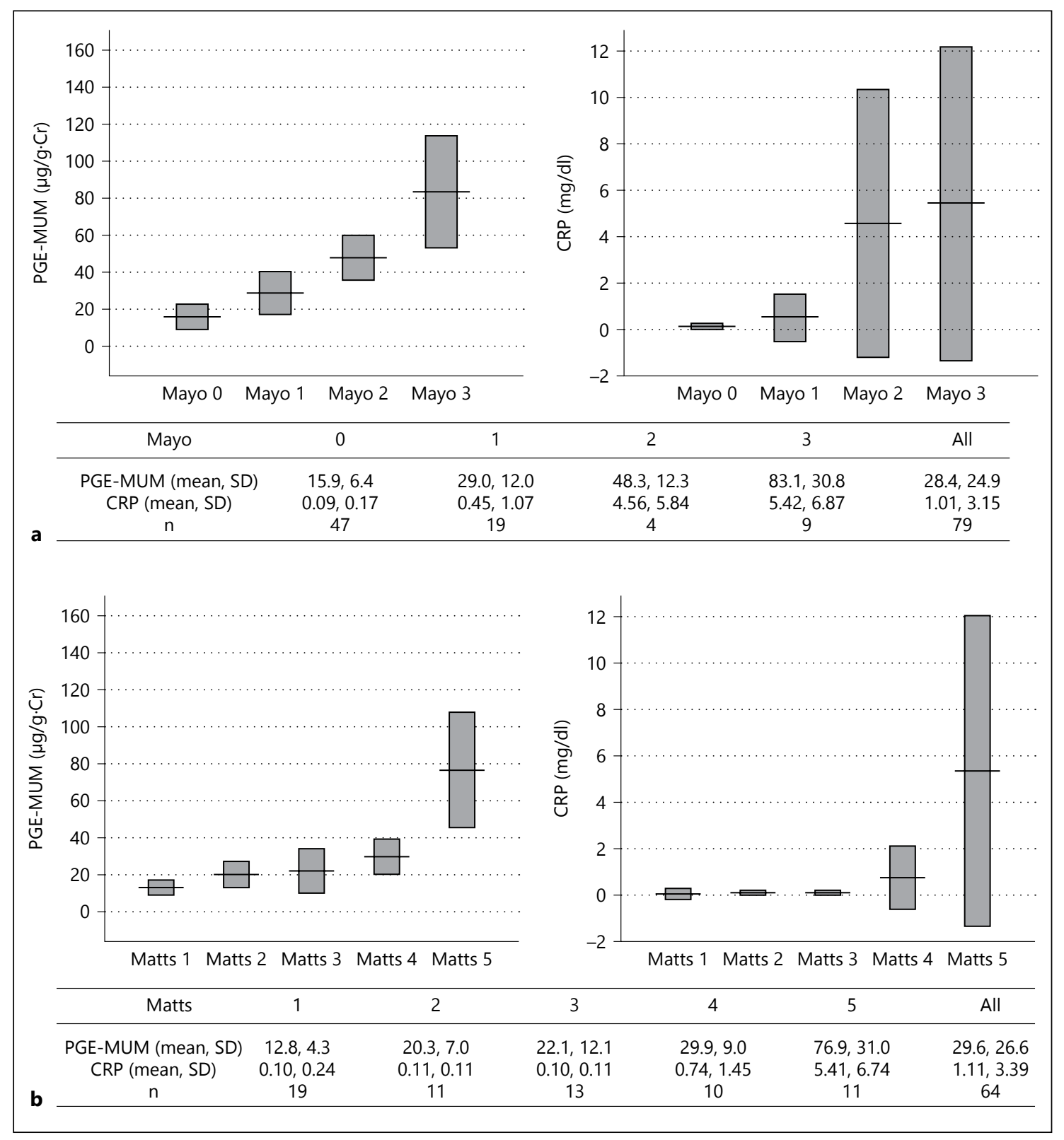

Fig. 2. Distribution of PGE-MUM and CRP levels based on colonoscopic and histological scores, and receiver operating characteristic curves for PGE-MUM and CRP in relation to clinical, colonoscopic, and histological activities (modified from [6]). a colonoscopic scores; b histological scores; c clinical activity; d colonoscopic activity; e histological activity. The PGE-MUM level was more sensitive than the CRP level in assessing UC activ-

CRP: $0.93>0.73,0.90>0.77$, and $0.89>0.75$, respectively; fig. 2). We think that mucosal healing should be confirmed by colonoscopic observation supported by histological assessment. Accordingly, the following examination and assessment were adopted. The most severe ity in cases of colonoscopic and histologic inflammation. AUC = Area under the curve; $\mathrm{C}=$ cutoff value; $\mathrm{S}=$ sensitivity (= specificity). In each row, lower activity is shown on the left and higher activity on the right. AUC is shown in each figure for PGE-MUM and CRP, respectively. + The value is greater than or equal to the adjacent number (e.g. $1+$ means $\geq 1$ ).

(For figure 2c-e see next page.)

instance of inflammation of the mucosa detected by total colonoscopy in each patient was used as the Mayo endoscopic score. We defined Mayo 0 as colonoscopic remission (= colonoscopically mucosal healing) and Mayo 1-3 as colonoscopically active disease. During the active phase 


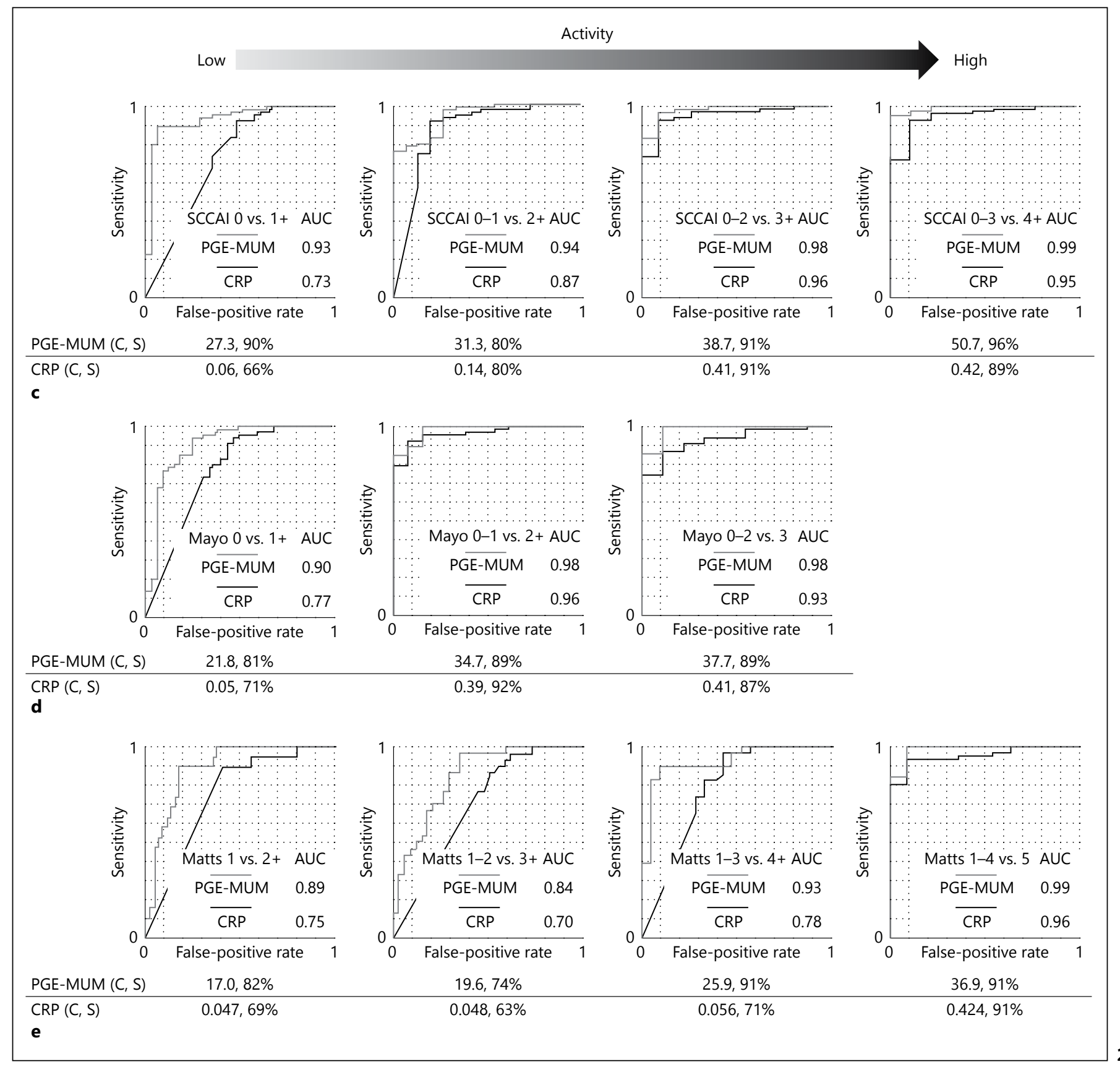

of UC, biopsy samples were taken of the most active inflammatory tissue viewed under colonoscopic observation. During the remission phase, non-inflammatory mucosal specimens of the rectum were obtained. The most severe instance of inflammation in each patient was used as the Matts biopsy grade. We defined Matts 1 as histologic remission (= histologically mucosal healing) and Matts $2-5$ as histologically active disease. Multivariate logistic regression analysis showed that PGE-MUM and
CRP are both useful for predicting remission of colonoscopic UC activity ( $p=0.02$ and $p=0.04$, respectively); however, only PGE-MUM was useful for predicting histological UC activity $(p<0.01)$. A cutoff value of 21.8 $\mu \mathrm{g} / \mathrm{g}$.Cr for PGE-MUM had a sensitivity of 0.81 for colonoscopic UC activity and remission, while a cutoff value of $17.0 \mu \mathrm{g} / \mathrm{g} \cdot \mathrm{Cr}$ had a sensitivity of 0.82 for histological UC activity and remission. Thus, with respect to sensitivity, PGE-MUM was superior to CRP as a biomarker of 
colonoscopic and histological UC activity that reflected mucosal healing.

The reason why CRP values remain in the normal range or show minor changes even in patients with active UC remains unknown. CRP is produced by hepatocytes following inflammatory stimulation, such as interleukin-6 (IL-6), tumor necrosis factor- $\alpha$, and interleukin- $1 \beta$. In patients with Crohn's disease (CD), disease activity is strongly correlated with CRP; however, its correlation with CRP in UC is weaker [3]. This may be due to the lower production of IL-6 in UC than in CD. However, the exact role of CRP in UC and CD remains unknown. The mechanisms of production of PGE-MUM and CRP upon inflammatory stimulation differ. PGE-MUM is believed to reflect the inflammatory condition of the intestine in UC more directly and accurately than CRP.

\section{Clinical Applications of PGE-MUM for UC Patients}

\section{Remission Phase of UC}

A mixture of colonoscopically active mucosa and remission mucosa is often seen in the clinical remission phase of UC. Although it is a phase of clinical and colonoscopic remission, the precise histological examination using biopsy samples obtained from each part of the whole colon occasionally shows active colitis partly $[6$, 24]. PGE-MUM levels can quantitatively indicate the inflammation level in the whole colonic mucosa. PGEMUM levels beyond $17 \mu \mathrm{g} / \mathrm{g}$. Cr would indicate the persistence of minor inflammation. In this situation, we recommend the inhibition of inflammation at the histological level. Suitable UC treatment based on the suppression of PGE2 production in the intestinal mucosa may lead to colonoscopic as well as histological remission, suppression of UC relapse, and prevention of colitis-associated cancer.

UC is a complex disease that requires professional advice and treatment, for which UC patients need to visit university hospitals and critical care medical centers. However, the number of patients diagnosed with UC has increased sharply in recent years, and general physicians are increasingly involved in treating UC patients along with professional gastroenterologists. Unfortunately, general physicians are handicapped by the lack of special instruments and diagnostic techniques; patients who require colonoscopic evaluation and/or biopsy require typically higher levels of care. However, we believe that general physicians can successfully use PGE-MUM levels for routine assessment of $\mathrm{UC}$ patients in the remission phase.

\section{Active Phase of UC}

In patients with highly active UC, high levels of PGEMUM and CRP are noted along with poor clinical scores. Such patients need to visit professional gastroenterologists or require hospital admission. However, in patients with intermediate UC activity, CRP levels may often remain normal despite the occurrence of diarrhea and/or abdominal pain [3]. In these patients, PGE-MUM levels would reflect the inflammation level more accurately and indicate the need for specialized or additional treatment. Thus, the measurement of PGE-MUM levels is expected to be highly useful for making clinical decisions. Although PGE-MUM may be a useful marker of UC activity, this measurement does not eliminate the need for colonoscopy and biopsy in the medical care of UC patients. Colonoscopy with a biopsy is required for the initial diagnosis of UC suspicious cases, and those with high risk of dysplasia with disease duration over 10 years.

\section{Conclusion}

PGE-MUM reflects intestinal mucosal inflammation in UC patients and may indicate colonoscopic and histological activity of UC. PGE-MUM test is a simple and noninvasive method that can detect faint levels of inflammation with good sensitivity and is useful for the evaluation of mucosal healing along with the treatment response, suggesting its usefulness as a biomarker of UC activity. This method can be used to reduce unnecessary colonoscopy and biopsy examinations, thereby reducing patient burden as well as lowering medical costs. While liquid chromatography/mass spectrometry for measuring PGE-MUM levels has been reported in several publications, the simple and easy radioimmunoassay method is more practical.

\section{Acknowledgments}

The authors thank the staff of the Jikei University School of Medicine for specimen collection and safekeeping. In particular, we thank Tomohiro Kato, Mika Matsuoka, Masayuki Saruta and Makoto Mitsunaga. We also greatly appreciate all of the staff of TFB Inc. and Fujirebio Inc. who performed specimen conveyance, processing, and data arrangement.

\section{Disclosure Statement}

The PGE-MUM measurement kit was provided by TFB Inc. (Tokyo, Japan). No other external funding was received.
38

Digestion 2016;93:32-39

DOI: $10.1159 / 000441665$
Arai/Matsuura/Matsuura/Fujiwara/ Okayasu/Ito/Arihiro 


\section{References}

1 Eaden JA, Abrams KR, Mayberry JF: The risk of colorectal cancer in ulcerative colitis: a meta-analysis. Gut 2001;48:526-535.

2 Beaugerie L, Itzkowitz SH: Cancers complicating inflammatory bowel disease. $\mathrm{N}$ Engl J Med 2015;372:1441-1452.

3 Vermeire S, Van Assche G, Rutgeerts P: Creactive protein as a marker for inflammatory bowel disease. Inflamm Bowel Dis 2004;10: 661-665.

4 Lauritsen K, Laursen LS, Bukhave K, et al: Effects of topical 5-aminosalicylic acid and prednisolone on prostaglandin E2 and leukotriene B4 levels determined by equilibrium in vivo dialysis of rectum in relapsing ulcerative colitis. Gastroenterology 1986;91:837-844.

5 Fujiwara M, Okayasu I, Oritsu M, et al: Significant increase in prostaglandin E-main urinary metabolite by laxative administration: comparison with ulcerative colitis. Digestion 2000;61:201-206

6 Arai Y, Arihiro S, Matsuura T, et al: Prostaglandin E-major urinary metabolite as a reliable surrogate marker for mucosal inflammation in ulcerative colitis. Inflamm Bowel Dis 2014;20:1208-1216.

7 Walsh A, Palmer R, Travis S: Mucosal healing as a target of therapy for colonic inflammatory bowel disease and methods to score disease activity. Gastrointest Endosc Clin N Am 2014;24:367-378.

8 Langhorst J, Lauche R: It is about time - tailoring of an individualized multimodal treatment approach in ulcerative colitis. Digestion 2014;89:139-141.

9 Furusawa Y, Obata Y, Fukuda S, et al: Commensal microbe-derived butyrate induces the differentiation of colonic regulatory $\mathrm{T}$ cells. Nature 2013;504:446-450

10 Fujiwara M, Kaneko T, Iwana H, et al: Inhibitory effects of Bifidobacterium longum on experimental ulcerative colitis induced in mice by synthetic dextran sulfate sodium. Digestion 2003;67:90-95.

11 Ohkusa T, Yoshida T, Sato N, et al: Commensal bacteria can enter colonic epithelial cells and induce proinflammatory cytokine secretion: a possible pathogenic mechanism of ulcerative colitis. J Med Microbiol 2009;58(pt 5):535-545.

12 Yoshida T, Sekine T, Aisaki K, et al: CITED2 is activated in ulcerative colitis and induces p53-dependent apoptosis in response to butyric acid. J Gastroenterol 2011;46:339349.

13 Zackular JP, Baxter NT, Iverson KD, et al: The gut microbiome modulates colon tumorigenesis. MBio 2013;4:e00692-e00713.
14 Arthur JC, Gharaibeh RZ, Muhlbauer M, et al: Microbial genomic analysis reveals the essential role of inflammation in bacteria-induced colorectal cancer. Nat Commun 2014;5:4724.

15 Okayasu I, Ohnishi H, Sarandi I, et al: Significant increase of prostaglandin E-major urinary metabolite in male smokers: a screening study of age and gender differences using a simple radioimmunoassay. J Clin Lab Anal 2014;28:32-41.

16 Milton-Thompson GJ, Cummings JH, Newman A, et al: Colonic and small intestinal response to intravenous prostaglandin F2 alpha and E2 in man. Gut 1975;16:42-46.

17 Sasaki S, Hirata I, Maemura K, et al: Prostaglandin E2 inhibits lesion formation in dextran sodium sulphate-induced colitis in rats and reduces the levels of mucosal inflammatory cytokines. Scand J Immunol 2000;51:23-28.

18 Xia D, Wang D, Kim SH, et al: Prostaglandin E2 promotes intestinal tumor growth via DNA methylation. Nat Med 2012;18:224226.

19 Yan M, Rerko RM, Platzer P, et al: 15-Hydroxyprostaglandin dehydrogenase, a COX-2 oncogene antagonist, is a TGF-beta-induced suppressor of human gastrointestinal cancers. Proc Natl Acad Sci U S A 2004;101:17468-17473.

20 Ishikawa $\mathrm{H}$, Mutoh $\mathrm{M}$, Suzuki S, et al: The preventive effects of low-dose enteric-coated aspirin tablets on the development of colorectal tumours in Asian patients: a randomised trial. Gut 2014;63:1755-1759.

21 Fink SP, Yamauchi M, Nishihara R, et al: Aspirin and the risk of colorectal cancer in relation to the expression of 15-hydroxyprostaglandin dehydrogenase (HPGD). Sci Transl Med 2014;6:233re2.

22 Okayasu I, Hatakeyama S, Yamada M, et al: A novel method in the induction of reliable experimental acute and chronic ulcerative colitis in mice. Gastroenterology 1990;98:694-702.

23 Okayasu I, Yamada M, Mikami T, et al: Dysplasia and carcinoma development in a repeated dextran sulfate sodium-induced colitis model. J Gastroenterol Hepatol 2002; 17: 1078-1083.

24 Okayasu I: Development of ulcerative colitis and its associated colorectal neoplasia as a model of the organ-specific chronic inflammation-carcinoma sequence. Pathol Int 2012; 62:368-380

25 Kawada N, Kristensen DB, Asahina K, et al: Characterization of a stellate cell activationassociated protein (STAP) with peroxidase activity found in rat hepatic stellate cells. J Biol Chem 2001;276:25318-25323.

26 Oleksiewicz U, Liloglou T, Tasopoulou KM, et al: Cytoglobin has bimodal: tumour suppressor and oncogene functions in lung cancer cell lines. Hum Mol Genet 2013;22:3207-3217.
27 Motoyama H, Komiya T, Thuy le TT, et al: Cytoglobin is expressed in hepatic stellate cells, but not in myofibroblasts, in normal and fibrotic human liver. Lab Invest 2014;94:192207

28 Okayasu I, Mikami T, Yoshida T, et al: Cytoglobin expression of rectal subepithelial myofibroblasts: significant alterations of cytoglobin+ stromal cells in long-standing ulcerative colitis. Histol Histopathol 2011;26:679-688.

29 Nishiguchi S, Kuroki T, Nakatani S, et al: Randomised trial of effects of interferon-alpha on incidence of hepatocellular carcinoma in chronic active hepatitis $\mathrm{C}$ with cirrhosis. Lancet 1995;346:1051-1055.

30 Tarao K, Rino Y, Ohkawa S, et al: Association between high serum alanine aminotransferase levels and more rapid development and higher rate of incidence of hepatocellular carcinoma in patients with hepatitis $\mathrm{C}$ virus-associated cirrhosis. Cancer 1999;86:589-595.

31 Mitsuhashi J, Mikami T, Saigenji K, et al: Significant correlation of morphological remodeling in ulcerative colitis with disease duration and between elevated p53 and p21 expression in rectal mucosa and neoplastic development. Pathol Int 2005;55:113-121.

32 Yoshida T, Mikami T, Mitomi H, et al: Diverse p53 alterations in ulcerative colitis-associated low-grade dysplasia: full-length gene sequencing in microdissected single crypts. J Pathol 2003;199:166-175.

33 Okayasu I, Hana K, Yoshida T, et al: Significant increase of colonic mutated crypts in ulcerative colitis correlatively with duration of illness. Cancer Res 2002;62:2236-2238.

34 Matsumoto N, Yoshida T, Okayasu I: High epithelial and stromal genetic instability of chromosome 17 in ulcerative colitis-associated carcinogenesis. Cancer Res 2003;63:61586161.

35 Okayasu I, Yoshida T, Mikami T, et al: Mucosal remodeling in long-standing ulcerative colitis with colorectal neoplasia: significant alterations of NCAM+ or alpha-SMA+ subepithelial myofibroblasts and interstitial cells. Pathol Int 2009;59:701-711.

36 Walmsley RS, Ayres RC, Pounder RE, et al: A simple clinical colitis activity index. Gut 1998; 43:29-32.

37 Schroeder KW, Tremaine WJ, Ilstrup DM: Coated oral 5-aminosalicylic acid therapy for mildly to moderately active ulcerative colitis. A randomized study. N Engl J Med 1987;317: 1625-1629.

38 Matts SG: The value of rectal biopsy in the diagnosis of ulcerative colitis. Q J Med 1961; 30:393-407.
PGE-MUM as a Biomarker for Inflammation in UC
Digestion 2016;93:32-39

DOI: $10.1159 / 000441665$ 